\title{
Adaptive Mesh and Algorithm Refinement Using Direct Simulation Monte Carlo
}

\author{
Alejandro L. Garcia, ${ }^{*}, 1$ John B. Bell, ${ }^{*}$ William Y. Crutchfield, ${ }^{*}$ and Berni J. Alder† \\ * Center for Computational Sciences and Engineering, Lawrence Berkeley National Laboratory, Berkeley, \\ California 94720; †Lawrence Livermore National Laboratory, Livermore, California 94550 \\ E-mail: garcia@mothra.lbl.gov,jbb@mothra.lbl.gov,wyc@mothra.lbl.gov, alder1@1lnl.gov
}

Received February 15, 1999; revised May 10, 1999

\begin{abstract}
Adaptive mesh and algorithm refinement (AMAR) embeds a particle method within a continuum method at the finest level of an adaptive mesh refinement (AMR) hierarchy. The coupling between the particle region and the overlaying continuum grid is algorithmically equivalent to that between the fine and coarse levels of AMR. Direct simulation Monte Carlo (DSMC) is used as the particle algorithm embedded within a Godunov-type compressible Navier-Stokes solver. Several examples are presented and compared with purely continuum calculations. (c) 1999 Academic Press
\end{abstract}

\section{INTRODUCTION}

When a large range of scales must be spanned, computational fluid dynamics (CFD) calculations often employ local mesh refinement so that a fine grid is used only in those regions that require high resolution. However, hydrodynamic formulations break down as the grid spacing approaches the molecular scale, for example, the mean free path in a gas. This paper describes adaptive mesh and algorithm refinement (AMAR), in which a continuum algorithm, such as a Navier-Stokes solver, is replaced by a particle algorithm, such as direct simulation Monte Carlo (DSMC), at the finest grid scale.

As an illustration, consider the flow of a gas through a microscopic channel, such as between the head and platter in a disk drive [1]. The continuum description of the flow and the quantities derived from it, such as wall drag, are not accurate whenever the Knudsen number $\mathrm{Kn}>10^{-2}$, where $\mathrm{Kn} \equiv \lambda / L, \lambda$ is the mean free path and $L$ is the channel width [2] Kinetic theory extensions to the continuum equations (e.g., Burnett expansion) have had limited success [3]. Another approach is to introduce kinetic corrections to the boundary conditions but these are often not accurate $[1,4,5]$ and can even give wrong qualitative features of the flow [6].

\footnotetext{
${ }^{1}$ Permanent address: Physics Department, San Jose State University, San Jose, CA 95192.
} 
Rigorously, a kinetic formulation is required at microscopic scales, however, at hydrodynamic scales the continuum approximation is valid. AMAR uses a particle method in regions of a flow requiring microscopic resolution and a continuum method, with varying levels of refinement, to evaluate the flow at larger scales. Thus, AMAR provides an effective methodology to span a broad range of length scales while retaining the advantages of a kinetic formulation where required.

At both rarefied and atmospheric densities the best particle method to use is direct simulation Monte Carlo. DSMC is several orders of magnitude more efficient than molecular dynamics for the simulation of gases; however, it remains several orders of magnitude less efficient than continuum CFD methods. For these reasons several researchers have investigated coupling the DSMC algorithm to a hydrodynamic solver. There exist loosely coupled schemes for which a continuum method provides a boundary condition for a DSMC code [7] or in which the two methods calculate different quantities in the problem (e.g., continuum method for the flow field and a particle method for the chemistry [8]). However, the focus here is on strongly coupled schemes where the DSMC and continuum methods simultaneously evaluate different regions of the flow and continuously exchange information across an interface.

Wadsworth and Erwin first demonstrated such a scheme in calculating a one-dimensional shock wave profile [9] and a two-dimensional slit flow [10]. Related hybrid schemes were developed by Eggers and Beylich [11], Bourgat et al. [12], Le Tallec and Mallinger [13], and the author $[14,15]$. Hash and Hassan performed detailed studies of a DSMC/Navier-Stokes hybrid using the Marshak condition for resolving fluxes [16]. They also demonstrated that a Chapman-Enskog distribution was required when the viscous fluxes were significant but that a simple Maxwellian distribution was adequate when the continuum region was well approximated by the Euler equations [7]. Special purpose continuum solvers, which are closely tied to kinetic theory, have been proposed for use in hybrid schemes. Specifically, the kinetic flux-vector splitting (KFVS) [17] and adaptive discrete velocity (ADV) Euler solver [18] have been tested.

While adaptive mesh and algorithm refinement may superficially resemble other hybrid schemes, it differs fundamentally from all of them. First, AMAR is specifically designed to work as a multi-level method for simulating systems whose length scales span several orders of magnitude. AMAR is a natural extension of adaptive mesh refinement (AMR) and can easily be implemented within an existing AMR code. Second, the AMAR coupling between the particle and continuum regions conserves mass, momentum, and energy to within roundoff error. Not only does this eliminate any systematic drift in the solution (e.g., mass loss in a closed system), it also improves the numerical stability of the method. Third, the continuum solver can easily be changed to any conservative (i.e., flux-based) scheme, either implicit or explicit. Only four basic subroutines, outlined at the end of Section 4, couple the continuum solver to the particle algorithm. Finally, some hybrids schemes are limited, in theory or in practice, to the simulation of one- or two-dimensional problems; AMAR is fully three dimensional.

This paper presents the framework for adaptive mesh and algorithm refinement and illustrates its use by incorporating a DSMC simulation at the finest level of an adaptive mesh refinement hierarchy. The DSMC algorithm and AMR scheme are briefly described in Sections 2 and 3. The AMAR technique for coupling these methods is presented in Section 4 and results from AMAR calculations in Section 5. Finally, Section 6 describes future work. 


\section{DIRECT SIMULATION MONTE CARLO}

Direct simulation Monte Carlo (DSMC) is a well-established algorithm for computing gas dynamics at the level of the Boltzmann equation. For completeness, this section presents a summary of the method, emphasizing those elements that are relevant to formulating AMAR. The DSMC algorithm is described in detail in [19]; see [20] for a tutorial and $[21,22]$ for reviews.

In DSMC, the state of the system is given by the positions and velocities of particles, $\left\{\mathbf{r}_{i}, \mathbf{v}_{i}\right\}$. First, the particles are moved as if they did not interact, that is, their positions are updated to $\mathbf{r}_{i}+\mathbf{v}_{i} \Delta t$. Any particles that reach a boundary are processed according to the appropriate boundary condition. Second, after all particles have moved, a given number are randomly selected for collisions. This splitting of the evolution between streaming and collisions is only accurate when the time step, $\Delta t$, is a fraction of the mean collision time for a particle.

The concept of "collision" implies that the interaction potential between particles is short-ranged. In the simulations presented here the particles are taken to be rigid spheres of diameter $\sigma$. Extensions to other representations of the molecular interaction may be used to give more realistic transport properties [19] and equations of state [23, 24]. For hard sphere particles, the number of collisions among $N$ particles in a cell volume $V$ during a time step is

$$
M=\frac{N^{2} \pi \sigma^{2}\left\langle v_{\mathrm{r}}\right\rangle \Delta t}{2 V},
$$

where $\left\langle v_{\mathrm{r}}\right\rangle$ is the average relative speed among the particles. Bird's "no time counter" method [19] for computing collision frequency is used since it avoids the explicit evaluation of $\left\langle v_{\mathrm{r}}\right\rangle$.

Particles are randomly selected as collision partners with the restriction that their mean separation be a fraction of a mean free path [25]. This restriction is enforced by ensuring that cell dimensions are less than a mean free path. For hard spheres, the probability of selecting a given pair is proportional to the relative speed between the particles. DSMC evaluates individual collisions stochastically, conserving momentum and energy and selecting the post-collision angles from their kinetic theory distributions. For hard spheres, the center of mass velocity and relative speed are conserved in the collision with the direction of the relative velocity uniformly distributed in the unit sphere. This Markov approximation of the collision process is statistically accurate so long as the number of particles in a collision cell is sufficiently large, typically over twenty [26, 27].

These constraints on time step, cell size, and number of particles make DSMC computationally expensive unless the physical domain is small or the gas is highly rarefied. For example, the efficiency of the method can be judged by the observation that a simulation of air at standard temperature and pressure requires about $10^{6}$ particles per cubic micron and $10^{4}$ time steps per microsecond.

\section{ADAPTIVE MESH REFINEMENT}

In a computational fluid dynamics calculation, the standard hydrodynamic variables are density $\rho$, fluid velocity $\mathbf{u}=\left[\begin{array}{lll}u_{x} & u_{y} & u_{z}\end{array}\right]$, and pressure $P$. From these one may obtain the conserved densities of mass $\rho$, momentum $\mathbf{p}$, and energy, $e$. The compressible NavierStokes equations may be written in the conservative form [28],

$$
\frac{\partial \mathbf{U}}{\partial t}+\nabla \cdot \mathbf{F}=\nabla \cdot \mathbf{D}
$$


where $\mathbf{U}$ is a vector composed of the conserved densities, $\mathbf{F}=\left(\mathbf{F}^{\mathbf{x}}, \mathbf{F}^{\mathbf{y}}, \mathbf{F}^{\mathbf{z}}\right)$ represent the hyperbolic flux terms, and $\mathbf{D}=\left(\mathbf{D}^{\mathbf{x}}, \mathbf{D}^{\mathbf{y}}, \mathbf{D}^{\mathbf{z}}\right)$ the parabolic flux terms. More precisely,

$$
\mathbf{U}=\left(\begin{array}{c}
\rho \\
p_{x} \\
p_{y} \\
p_{z} \\
e
\end{array}\right) ; \quad \mathbf{F}^{\mathbf{x}}=\left(\begin{array}{c}
\rho u_{x} \\
\rho u_{x}^{2}+P \\
\rho u_{x} u_{y} \\
\rho u_{x} u_{z} \\
(e+P) u_{x}
\end{array}\right) ; \quad \mathbf{D}^{\mathbf{x}}=\left(\begin{array}{c}
0 \\
\tau_{x x} \\
\tau_{x y} \\
\tau_{x z} \\
u_{i} \tau_{x i}-q_{x}
\end{array}\right)
$$

where $\tau$ and $q$ are the stress tensor and heat flux, respectively, with similar expressions for the other flux terms.

In the AMAR methodology presented here, the compressible Navier-Stokes equations are integrated using a second-order unsplit Godunov method to evaluate the hyperbolic fluxes [29] and a standard finite difference approximation using Crank-Nicolson temporal differencing to treat the parabolic terms. Thus, the discretization has the form

$$
\begin{aligned}
& \frac{\mathbf{U}_{i j k}^{n+1}-\mathbf{U}_{i j k}^{n}}{\Delta t}+\frac{\mathbf{F}_{i+\frac{1}{2}, j, k}^{x, n+\frac{1}{2}}-\mathbf{F}_{i-\frac{1}{2}, j, k}^{x, n+\frac{1}{2}}}{\Delta x}+\frac{\mathbf{F}_{i, j+\frac{1}{2}, k}^{y, n+\frac{1}{2}}-\mathbf{F}_{i, j-\frac{1}{2}, k}^{y, n+\frac{1}{2}}}{\Delta y}+\frac{\mathbf{F}_{i, j, k+\frac{1}{2}}^{z, n+\frac{1}{2}}-\mathbf{F}_{i, j, k-\frac{1}{2}}^{z, n+\frac{1}{2}}}{\Delta z} \\
& =\frac{1}{2}\left(\frac{\mathbf{D}_{i+\frac{1}{2}, j, k}^{x, n+1}+\mathbf{D}_{i+\frac{1}{2}, j, k}^{x, n}-\mathbf{D}_{i-\frac{1}{2}, j, k}^{x, n+1}-\mathbf{D}_{i-\frac{1}{2}, j, k}^{x, n}}{\Delta x}\right. \\
& +\frac{\mathbf{D}_{i, j+\frac{1}{2}, k}^{y, n+1}+\mathbf{D}_{i, j+\frac{1}{2}, k}^{y, n}-\mathbf{D}_{i, j-\frac{1}{2}, k}^{y, n+1}-\mathbf{D}_{i, j-\frac{1}{2}, k}^{y, n}}{\Delta y} \\
& \left.+\frac{\mathbf{D}_{i, j, k+\frac{1}{2}}^{z, n+1}-\mathbf{D}_{i, j, k+\frac{1}{2}}^{z, n}-\mathbf{D}_{i, j, k-\frac{1}{2}}^{z, n+1}-\mathbf{D}_{i, j, k-\frac{1}{2}}^{z, n}}{\Delta z}\right) .
\end{aligned}
$$

The implicit discretization of the parabolic terms requires the solution of a nonlinear system of equations which is easily treated using standard nonlinear multigrid ideas. The computation of the hyperbolic flux terms using the second-order Godunov procedure is an explicit procedure so that the integration algorithm has a time step restriction based on CFL considerations for the Euler equations $(\mathbf{D}=0)$.

For problems in fluid dynamics where there are a large range of scales that must be spanned, some form of adaptive mesh refinement is used to localize high resolution to the areas where it is required. In the AMR methodology, a block-structured hierarchical form of refinement, first developed by Berger and Oliger [30] for hyperbolic partial differential equations, is used. A conservative version of this methodology for gas dynamics was developed by Berger and Colella [31] and extended to three dimensions by Bell et al. [32].

AMR is based on a sequence of nested levels of refinement with successively finer spacing in both time and space. In this approach, fine grids are formed by dividing coarse cells by a refinement ratio, $r$, in each direction. Increasingly finer grids are recursively embedded in coarse grids until the solution is adequately resolved with each level contained in the next coarser level. An error estimation procedure based on user-specified criteria evaluates where additional refinement is needed and grid generation procedures dynamically create or remove rectangular fine grid patches as resolution requirements change.

The adaptive time-step algorithm advances grids at different levels using time steps appropriate to that level based on CFL considerations. The time-step procedure can most 
easily be thought of as a recursive algorithm, in which to advance level $l$ (level $l=0$ being the coarsest and $l=l_{\max }$ the finest), the following steps are taken:

- Advance level $l$ in time as if it is the only level. Supply boundary conditions for $\mathbf{U}$ from level $l-1$ if level $l>0$, and from the physical boundary conditions.

- If $l<l_{\max }$

-Advance level $(l+1) r$ times with time step $\Delta t^{l+1}=\frac{1}{r} \Delta t^{l}$ using boundary conditions for $\mathbf{U}$ from level $l$, and from the physical domain boundaries.

-Synchronize the data between levels $l$ and $l+1$, and interpolate corrections to higher levels if $l+1<l_{\max }$.

The adaptive algorithm, as outlined above, performs operations to advance each level independent of other levels in the hierarchy (except for boundary conditions) and then computes a correction to synchronize the levels. Loosely speaking, the objective in this synchronization step is to compute the modifications to the coarse grid that reflect the change in the coarse grid solution from the presence of the fine grid. There are two steps in the synchronization. First, the fine grid is averaged onto the coarse grid; i.e., the conserved quantities on coarse grid cells covered by fine grid are replaced by the average of the fine grid.

The second step of the synchronization, called "refluxing," corrects for the difference in coarse and fine grid fluxes at the boundary of the fine grid. The basic approach used here is an analog of the procedure used by Almgren et al. [33] extended to the case of nonlinear parabolic terms. During the course of the integration step, flux information is saved at the faces on the boundary of the coarse and fine grid to obtain the difference between the fluxes calculated at level $l$ and the corresponding level $l+1$ average. The latter are the fluxes at level $l+1$ time averaged over the level $l$ time step and spatially averaged over the area of the level $l$ face. This time step- and area-weighted flux difference is

$$
\begin{aligned}
\delta \mathcal{F}^{l}= & \Delta t^{l}\left(-A^{l}\left(\mathbf{F}^{n+\frac{1}{2}, l}-\frac{1}{2}\left(\mathbf{D}^{n, l}+\mathbf{D}^{n+1, l}\right)\right)\right. \\
& \left.+\frac{1}{r} \sum_{k=0}^{r-1} \sum_{\text {faces }} A^{l+1}\left(\mathbf{F}^{r n+k+\frac{1}{2}, l+1}-\frac{1}{2}\left(\mathbf{D}^{r n+k, l+1}+\mathbf{D}^{r n+k+1, l+1}\right)\right)\right),
\end{aligned}
$$

where $\mathbf{F}$ and $\mathbf{D}$ are the components of the convective and diffusive fluxes corresponding to the faces in Eq. (4) and $A$ is the signed area of the face of a grid cell where the sign depends on the direction normal to the face, facing away from the fine grid. The sum over faces in Eq. (5) is a sum over all fine grid faces that cover the coarse face.

The flux correction, $\delta \mathcal{F}_{n}^{l}$, represents the difference between the flux used to update the coarse cells adjacent to the fine grid and the fluxes that are computed on the fine grid. To ensure stability for low Reynolds numbers and to match the implicit, Crank-Nicolson character of the diffusive step an implicit solve is performed

$$
\delta \mathbf{U}-\frac{\Delta t}{2} \nabla \cdot \mathbf{D}\left(\overline{\mathbf{U}^{n+1}}+\delta \mathbf{U}\right)=\frac{\Delta t \delta \mathcal{F}}{\Delta x \Delta y \Delta z},
$$

where $\overline{\mathbf{U}^{n+1}}$ is the coarse grid solution after averaging the fine grid solution but before computing the correction. The coarse grid, $\mathbf{U}^{n+1}$, is updated by

$$
\mathbf{U}^{n+1}=\overline{\mathbf{U}^{n+1}}+\delta \mathbf{U}
$$


The fine grid is updated by using a conservative scheme that interpolates the correction to the fine grid and to other finer grids contained within the fine grid. Finally, to capture the effect of the synchronization of level $l$ and higher on level $l-1$ the flux corrections $\mathcal{F}^{l-1}$ are not updated until after the synchronization of levels $l$ and $l+1$.

\section{ADAPTIVE MESH AND ALGORITHM REFINEMENT}

Adaptive mesh and algorithm refinement (AMAR) uses the same basic algorithmic outline as AMR, as presented in the previous section, except that the finest grid level is evaluated by a DSMC calculation. For the purposes of exposition, a DSMC region is considered embedded within a single-level continuum grid.

At the start of a continuum time step, fluxes are computed at each cell face and used to advance the conserved densities (mass, momentum, and energy) on the grid. All continuum cells are advanced by $\Delta t_{\text {cont }}$, including those that overlay the DSMC region. For numerical stability, $\Delta t_{\text {cont }}=C \Delta x /|c+v|$ where $c$ is the sound speed, $v$ is the maximum fluid speed, and $C<1$ is the Courant number. Next, the particle calculation advances to the same time by taking several, smaller time steps, $\Delta t_{\text {part }}$. Though $\Delta t_{\text {part }}$ is a fraction of the mean collision time, for the finest continuum grid $\Delta x \approx \lambda$ so $\Delta t_{\mathrm{c}} \approx \Delta t_{\text {part }}$. For the AMAR calculations presented in this paper, the width of the smallest continuum cells is two mean free paths and $\Delta t_{\text {cont }}<4 \Delta t_{\text {part }}$.

The DSMC region is surrounded by buffer cells (see Fig. 1). At the beginning of each DSMC time step, particles are created within the buffer cells according to the hydrodynamic values (density, fluid velocity, temperature) and their gradients on the overlaying continuum grid. Since the continuum grid advances first, these values are time interpolated between continuum time steps as DSMC is subcycled to reach the same time

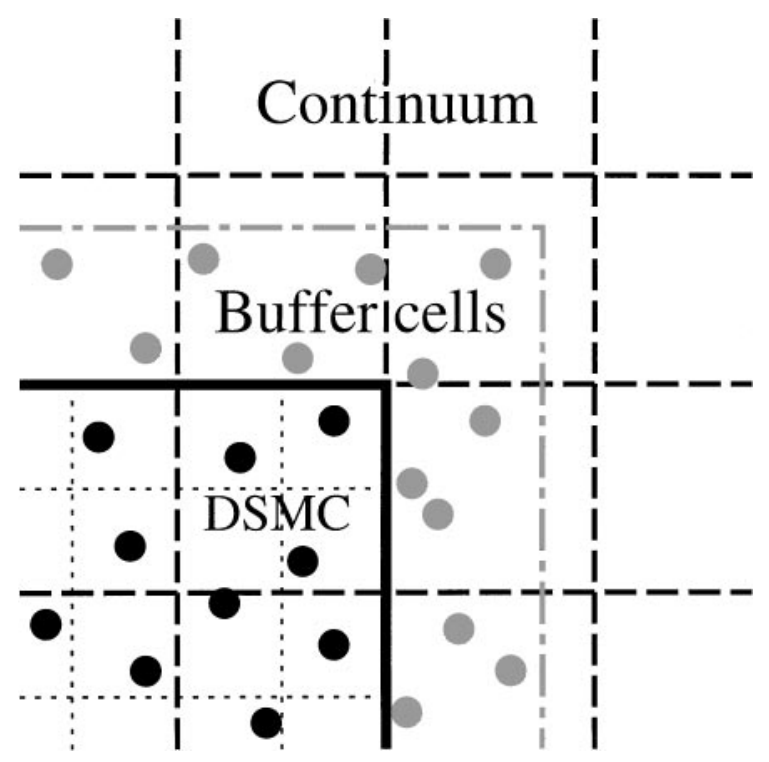

FIG. 1. Schematic showing a DSMC region and its surrounding buffer cells embedded within a continuum mesh. Continuum cells (dashed lines); DSMC collision cells (dotted lines); DSMC/continuum interface (solid line); buffer cell sheath (dot-dashed line). 
as the continuum solver. Buffer cells do not need to be entirely filled with particles. Only particles within a sheath near the DSMC region are generated with the thickness of this sheath determined adaptively. The particle velocities are drawn from the appropriate distribution for the continuum solver: the Maxwell-Boltzmann distribution for the Euler equations and the Chapman-Enskog distribution [34] for the Navier-Stokes equations.

Next, particles in both the main and buffer regions move a single DSMC time step. If a particle crosses the interface between these regions, that particle contributes to the flux for the coarse grid face through which it passes. The contribution of all particles crossing a coarse grid face during the DSMC steps plays the same role as the sum over fine-grid continuum grid faces in Eq. (5). After moving the particles, those remaining in or those that moved into the buffer region are discarded and collisions among the remaining particles are evaluated. The cell structure used in evaluating DSMC collisions is separate from and independent of the continuum grid.

A technical but important issue that any particle/continuum hybrid must confront is the "corner problem." Specifically, when a particle passes into the continuum region, it changes the mass, momentum, and energy density in a continuum cell. In AMAR, this particle's contribution is formulated as a flux on the cell side that lies on the interface between the continuum and particle regions. A similar flux contribution arises when a particle created within the buffer region crosses this interface. For example, in Fig. 2, when particle 1 passes from cell A to D (or vice versa), it contributes to the flux on the side between these cells. A more complicated case occurs when particle 2 passes from cell B to D. AMAR updates the flux on the side between cells B and E since that is where the particle crosses the interface. Finally, consider particle 3, which passes near the corner traveling from cell B to F. This particle contributes to the flux between cells B and C. There cannot be a flux contribution for cell $\mathrm{F}$ since it has no side bordering the particle/continuum interface. This last example illustrates that fluxes must be evaluated where a particle crosses the interface and not from the cell that the particle moves into. If a simulation does not handle the corners correctly, steady state flows can exhibit a spurious drift, such as loss of mass in a closed system.

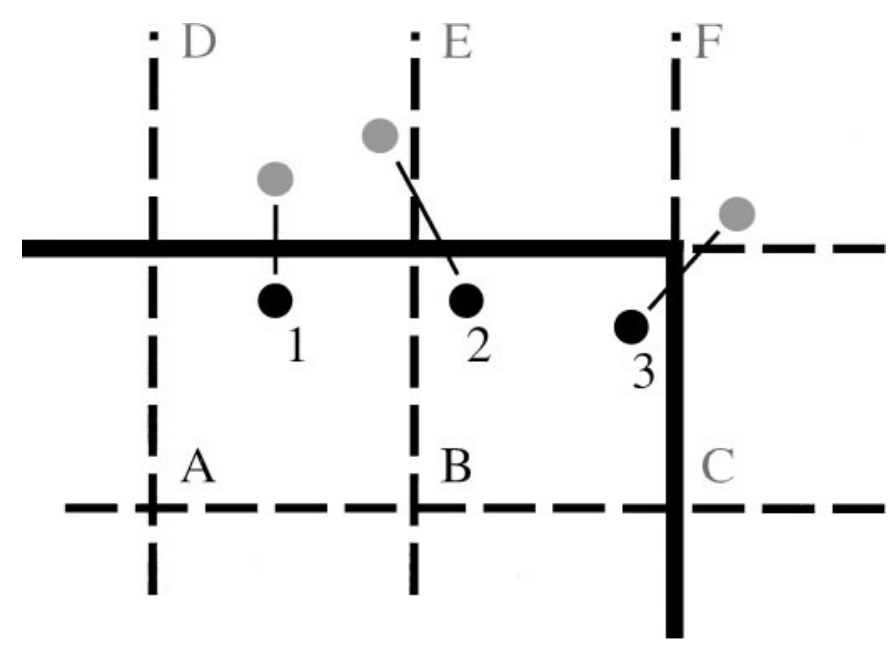

FIG. 2. Particles and continuum cells near a corner of the DSMC/continuum interface. 
When the DSMC region has advanced for an entire continuum grid time step, two synchronizations are performed, analogous to the AMR case described above. First, the continuum cells that overlay the central DSMC region are reset according to the conserved densities computed from the set of particles within each continuum grid cell. Second, Eq. (6) is solved to obtain a correction to the fluxes for the continuum grid using as right hand side

$$
\delta \mathcal{F}^{l}=\Delta t^{l}\left(-A^{l}\left(\mathbf{F}^{n+\frac{1}{2}, l}-\frac{1}{2}\left(\mathbf{D}^{n, l}-\mathbf{D}^{n+1, l}\right)\right)\right)+\sum_{p} \mathcal{F}_{p}
$$

where the sum represents the flux of the conserved quantities through carried by particles $p$ passing through the coarse face during the DSMC updates. Equation (7) is used to update the conserved quantities on the coarse grid.

From the implicit solve in Eq. (6), the values of $\delta \mathbf{U}$ on coarse cells covered by the DSMC region generate a correction $\delta \mathbf{p}$ and $\delta e$ in the momenta and energy for DSMC cells; there is no mass correction because mass does not diffuse. The velocity of each particle within a given continuum cell is corrected as

$$
\mathbf{v}^{\prime}=\left\langle\mathbf{v}^{\prime}\right\rangle+\alpha(\mathbf{v}-\langle\mathbf{v}\rangle)
$$

where $\left\langle\mathbf{v}^{\prime}\right\rangle=\langle\mathbf{v}\rangle+\delta \mathbf{p} / \rho$,

$$
\alpha=\left(1+\frac{\delta e+e_{k}-e_{k}^{\prime}}{e-e_{k}}\right)^{1 / 2}
$$

with $e_{k}=\frac{1}{2} \rho|\langle\mathbf{v}\rangle|^{2}, e_{k}^{\prime}=\frac{1}{2} \rho\left|\left\langle\mathbf{v}^{\prime}\right\rangle\right|^{2}$, and $e=\frac{1}{2} \rho\left\langle|\mathbf{v}|^{2}\right\rangle$; the angle brackets indicate averages over particles within a continuum cell. These synchronization steps guarantee that, in the absence of external sources, total mass, momentum, and energy are conserved to within round-off error in the computational domain. Note that when an explicit solver is used, as with the Euler equations, the correction is localized to the coarse cells adjacent to the DSMC region so no correction to the particles is required.

In summary, the interaction between the continuum solver and the DSMC region is encapsulated into four routines: (1) Passing the time-interpolated state to the particle buffer cells; (2) Passing the momentum and energy corrections, as computed in the implicit linear solve, to the DSMC region; (3) Receiving the fluxes recorded when particles cross the DSMC interface; (4) Receiving conserved densities for continuum cells overlaying the DSMC region. This coupling of the continuum grid with DSMC makes the latter appear just like any level of refinement in the purely continuum case.

\section{NUMERICAL EXAMPLES}

This section describes a series of numerical experiments that were performed to test and demonstrate the adaptive mesh and algorithm refinement framework. In each case a single DSMC region is embedded within a continuum grid on which either the Euler or NavierStokes equations are computed. In general, the particles in the buffer regions are generated using the Chapman-Enskog distribution [34]. However, for the purpose of comparison, when the Euler equations are used, both the Maxwell-Boltzmann and Chapman-Enskog distributions are considered. In the last example (flow past a sphere) the continuum solver 
uses two grid levels so the DSMC region is embedded within a fine grid which is itself embedded in a coarse grid. For all other examples, a single continuum grid is used.

The particles are treated as hard spheres of diameter $\sigma=0.366 \mathrm{~nm}$ and mass $m=6.63 \times$ $10^{-23} \mathrm{~g}$ (argon parameters). The reference density is $\rho_{0}=1.78 \times 10^{-3} \mathrm{~g} / \mathrm{cm}^{3}$; the mean free path at this density is $\lambda_{0}=62.5 \mathrm{~nm}$. The reference temperature is $T_{0}=273 \mathrm{~K}$ and the reference sound speed is $c_{0}=3.08 \times 10^{4} \mathrm{~cm} / \mathrm{s}$. The equation of state is the ideal gas law, $P=\rho k T / m$, where $k=1.3806 \times 10^{-23} \mathrm{~J} / \mathrm{K}$ is Boltzmann's constant. The viscosity and thermal conductivity are

$$
\mu=\frac{5}{16 d^{2}} \sqrt{\frac{m k T}{\pi}} ; \quad \kappa=\frac{15 k}{4 m} \mu,
$$

as given by the Chapman-Enskog theory.

All the simulations are fully three dimensional with at least 16 continuum grid cells in each direction. When a single grid is used, these cells are cubes of length $\Delta x=2 \lambda_{0}$; when there are two continuum levels, the cells are cubes of length $\Delta x$ and $2 \Delta x$. The reference CFL time step is $\Delta t_{0}=\Delta x / c_{0}=4.06 \times 10^{-10} \mathrm{~s}$ and the Courant number is 0.25 for all of the runs. With this Courant number the DSMC region typically performs from one to four time steps for each continuum time step.

At the reference density, the DSMC region contains 100 particles per $\lambda_{0}^{3}$. The collision frequency is computed using cubic cells of length $1.0 \lambda_{0}$, collision partners are selected within cubic subcells of length $0.5 \lambda_{0}$, and statistical samples are measured in cubes of length $0.8 \lambda_{0}$. The total number of particles in the various cases ranges from $5 \times 10^{4}$ to $6 \times 10^{6}$.

\subsection{Thermodynamic Equilibrium}

The simplest test case is thermodynamic equilibrium: the system is initially at rest with constant density and temperature. The continuum grid is $32 \times 32 \times 32$ with periodic boundary conditions on all sides. The DSMC region is a cube embedded in the continuum solver within the $4 \times 4 \times 4$ cells at the center of the system. Although the system is initially uniform, DSMC produces spontaneous fluctuations with the correct equilibrium spectrum [35]. After 2000 continuum time steps, the total mass in the simulation is conserved to better than one part in $10^{6}$ and the total energy to better than one part in $10^{5}$.

When the continuum solver employs the Navier-Stokes equations, the system remains in thermodynamic equilibrium. However, when the Euler equations are used in the continuum region, the number of particles in the DSMC region slowly increases and the energy density decreases so that thermodynamic equilibrium is not maintained. Since total mass, momentum, and energy are strictly conserved (to within round-off error), the mass in the continuum region decreases and the energy density increases. This rise in the number of DSMC particles as a function of time is shown in Fig. 3. While the Navier-Stokes/AMAR preserves the correct average, after 2000 steps the number of DSMC particles in the Euler/AMAR increases by $1.1 \%$ when using the Maxwell-Boltzman distribution in the buffer region and by $0.7 \%$ when using the Chapman-Enskog distribution. This error is reduced when the simulation uses more particles per cubic mean free path. For the runs displayed in Fig. 3 each continuum cell that overlays the DSMC region contains, on average, 800 particles.

The anomalous drift away from thermodynamic equilibrium that is observed using the Euler equations is not a flaw in the AMAR methodology. Other DSMC/Euler coupling 


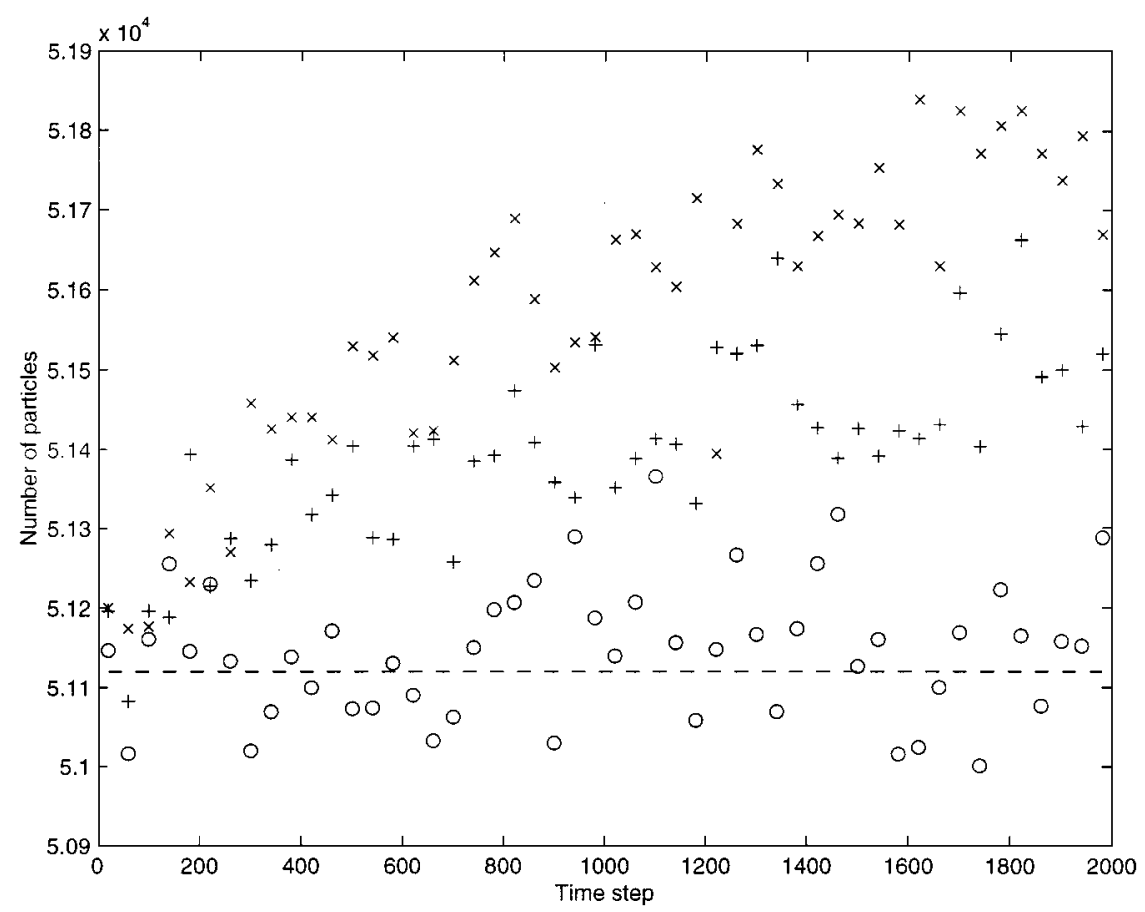

FIG. 3. Number of particles in the DSMC region versus time step for thermodynamic equilibrium. Euler AMAR using Maxwell-Boltzmann (×), Chapman-Enskog (+) compared with the Navier-Stokes AMAR (O) which varies about the initial value (dashed line).

schemes also exhibit this drift at equilibrium [36, 37]. The effect is due to the fact that fluctuations in the DSMC region transmit thermal and mechanical energy to the continuum region while only mechanical energy is returned since the Euler equations have no heat flux even when a temperature gradient is present. Thus the thermal energy in the continuum region rises and the density falls so as to maintain mechanical equilibrium (i.e., constant pressure). This spurious effect is masked when there is a net flow across the system. If the simulation is initialized with a uniform fluid speed of $0.05 c_{0}$, after 2000 time steps the number of DSMC particles in the Euler AMAR increases by $0.6 \%$ when using the MaxwellBoltzman distribution in the buffer region and by $0.4 \%$ when using the Chapman-Enskog distribution.

\subsection{Impulsive Piston}

The first non-equilibrium case considered is a gas initially at the reference density and temperature and moving at Mach 2 toward a thermal wall held at fixed temperature (Fig. 4a). This is equivalent to an impulsively started piston traveling into a gas initially at rest, in the reference frame of the piston. A normal shock develops in front of the wall and the shock front moves into the gas. Basic shock relations [38] give a shock speed of Mach 3, a density ratio of 3 across the shock, and a temperature ratio of $11 / 3$. The reference temperature in the undisturbed gas is $273 \mathrm{~K}$ so the wall temperature is fixed at $1001 \mathrm{~K}$. The boundary condition on the opposite side of the system is a plane of reflection symmetry. The flow near that boundary (a rarefaction fan) does not affect the shock wave within the time of the simulation and thus is not analyzed. Periodic boundary conditions are applied in the other directions. 

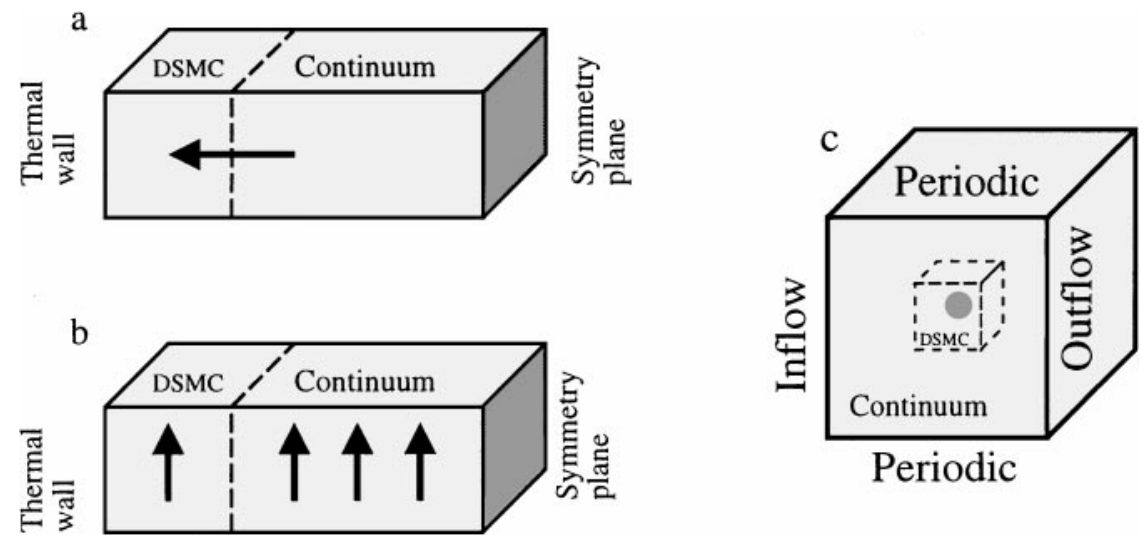

FIG. 4. Geometry for (a) impulsively started piston; (b) Rayleigh problem; (c) flow past a sphere.

The continuum grid contains $100 \times 16 \times 16$ cells leading to a system of length $12500 \mathrm{~nm}$, width and depth of $2000 \mathrm{~nm}$. The DSMC region, located next to the wall where the shock forms, has width and depth of $2000 \mathrm{~nm}$ and length of either 625 or $1250 \mathrm{~nm}$, equivalent to 5 or 10 continuum cells. In the larger DSMC simulation the number of particles is initially $2 \times 10^{6}$ and finally $6 \times 10^{6}$ when the shock passes into the continuum region. For comparison, a calculation with only the Navier-Stokes solver and no DSMC region is also considered.

Figures 5 and 6 show the temperature and density profiles near the piston wall at $t=2 \times 10^{-9} \mathrm{~s}$. For the AMAR run with the 5 cell DSMC region, the shock is just passing out of the particle region and it is in the center of the region for the 10 cell DSMC run. The latter run is practically equivalent to a purely DSMC simulation with fixed reservoirs since the values in the Navier-Stokes region are constant. The AMAR runs are in good agreement with each other; as expected, the temperature profile in the entire continuum run

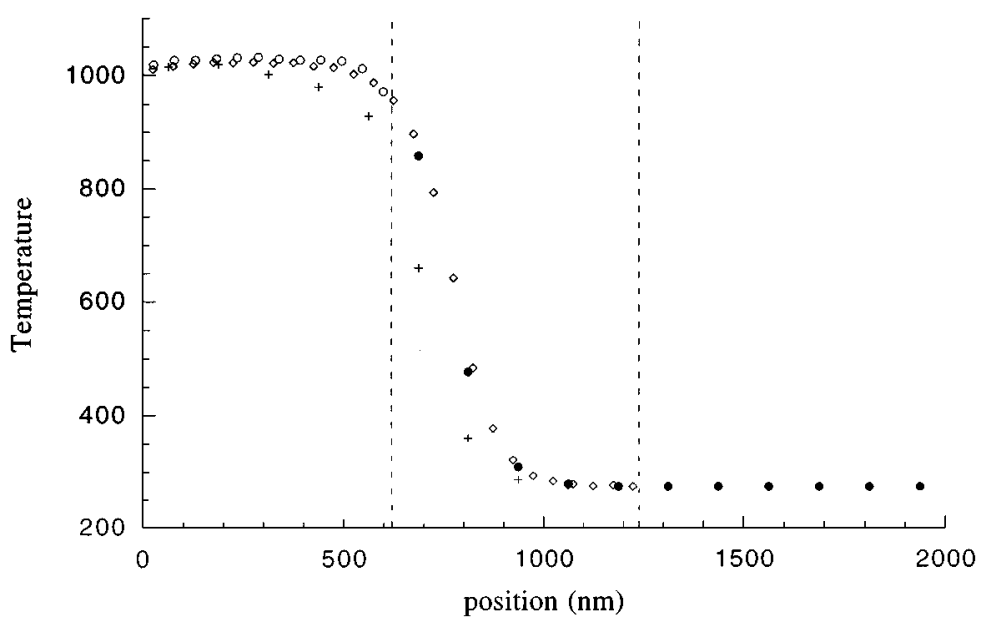

FIG. 5. Temperature versus position for the impulsively started piston at $t=2 \times 10^{-9} \mathrm{~s}$. AMAR run with 5-cell DSMC region $(\bigcirc)$; AMAR run with 10-cell DSMC region $(\diamond)$; and purely continuum with no DSMC run (+). Open symbols, DSMC data; filled symbols, AMAR continuum data. Dashed lines indicate the location of the particle/continuum interface for the AMAR runs. 


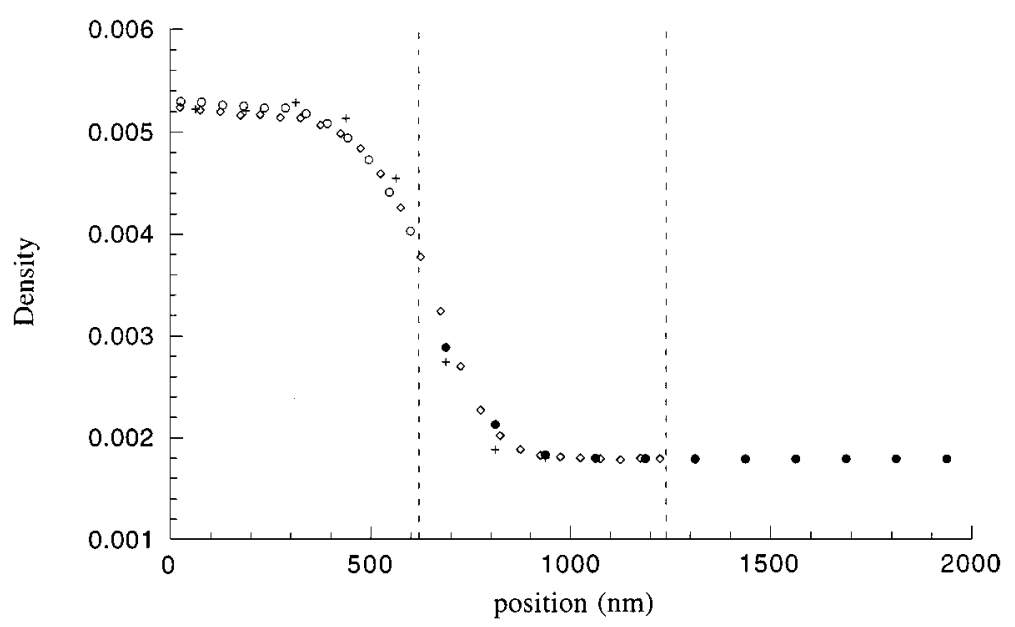

FIG. 6. Density versus position for the impulsively started piston at $t=2 \times 10^{-9} \mathrm{~s}$. See Fig. 5 for legend.

lags the DSMC data while the density profile is in better agreement [19]. The temperature and density profiles near the piston wall at $t=4 \times 10^{-9} \mathrm{~s}$ are shown in Figs. 7 and 8 . The temperature profiles of the two AMAR runs are again in good agreement while the density profiles are in fair agreement.

The impulsive piston is a severe test of the AMAR method since it is well known that the Navier-Stokes equations do not accurately predict the profiles of strong shocks. This is because the Chapman-Enskog expansion, on which the equations are based, breaks down when the characteristic length scale of hydrodynamic gradients is comparable to the mean free path. A breakdown parameter for the Chapman-Enskog distribution [34] is defined as $B=\max \left\{\left|\tau_{i j}^{*}\right|,\left|q_{i}^{*}\right|\right\}$, where

$$
\tau_{i j}^{*}=\frac{\mu}{P}\left(\frac{\partial u_{i}}{\partial x_{j}}+\frac{\partial u_{j}}{\partial x_{i}}-\frac{2}{3} \frac{\partial u_{k}}{\partial x_{k}} \delta_{i j}\right)
$$

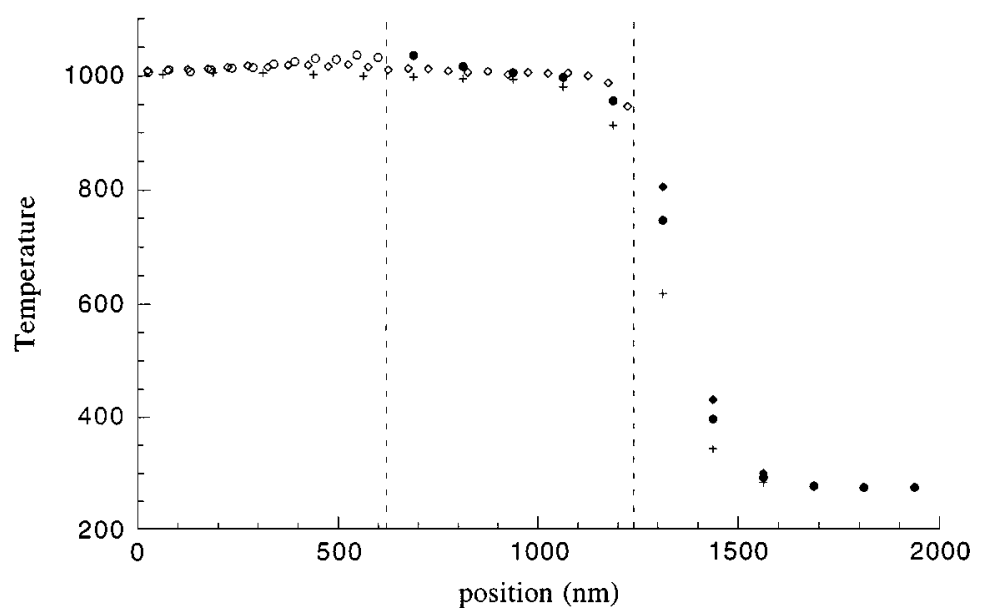

FIG. 7. Temperature versus position for the impulsively started piston at $t=4 \times 10^{-9} \mathrm{~s}$. See Fig. 5 for legend. 


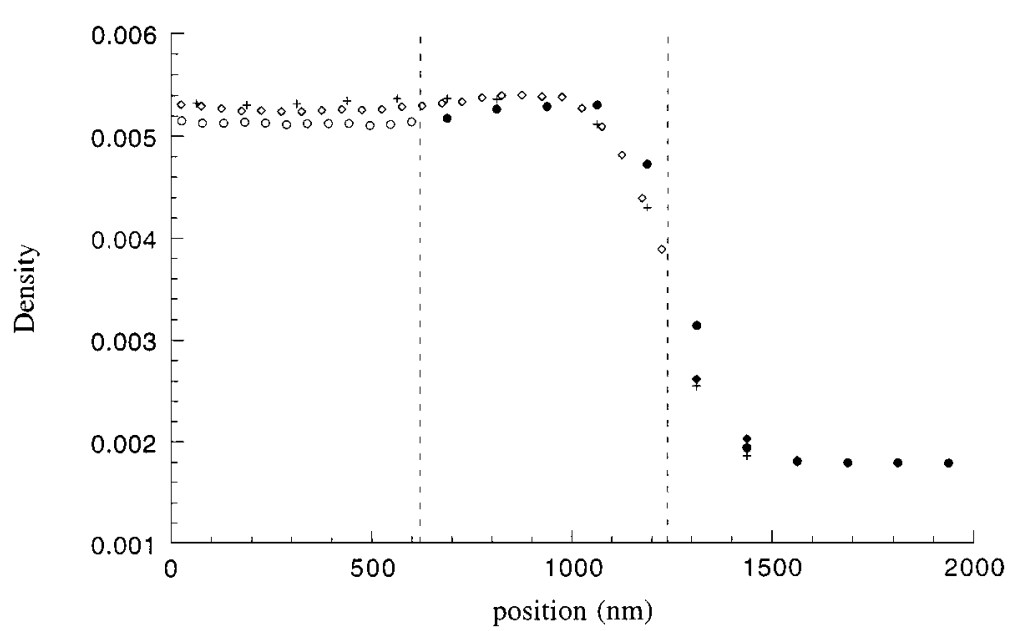

FIG. 8. Density versus position for the impulsively started piston at $t=4 \times 10^{-9} \mathrm{~s}$. See Fig. 5 for legend.

and

$$
q_{i}^{*}=-\frac{\kappa}{P}\left(\frac{2 m}{k T}\right)^{1 / 2} \frac{\partial T}{\partial x_{i}}
$$

are the normalized stress tensor and heat flux, respectively. This parameter is computed when the particle velocities are generated in the buffer regions; the validity of the distribution is questionable when $B>0.2$. In the AMAR simulations of the impulsive piston, the maximum value of $B$ was 1.3-1.4. For comparison, in the simulations of thermodynamic equilibrium $B$ is not zero due to spontaneous fluctuations yet it did not exceed 0.13 .

For the impulsive piston, the Euler AMAR program produces results similar to those presented above. The Euler equations are adequate for this flow since the advective fluxes are much greater than the dissipative fluxes. The main differences are that the shock thickness depends on the solver's numerical viscosity and that outside the DSMC region the density and temperature profiles of the shock front overlap. These differences between the Euler and Navier-Stokes solutions exist independent of whether or not the simulation includes a DSMC region.

\subsection{Rayleigh Problem}

This problem concerns a gas initially at the reference density and temperature moving at Mach 2 parallel to a stationary wall held at the reference temperature (Fig. 4b). This is the Rayleigh problem of an impulsively started wall shearing a gas initially at rest, as viewed in the reference frame of the moving wall. In time, the wall drags the gas to match its speed and the resulting velocity gradient produces viscous heating near the wall. The boundary condition on the opposite side is a plane of reflection symmetry; for short times the gas near this boundary remains undisturbed. Periodic boundary conditions are applied in the other directions.

The continuum grid contains $100 \times 16 \times 16$ cells, corresponding to a system of length $12,500 \mathrm{~nm}$ with width and depth of $2000 \mathrm{~nm}$. The DSMC region, located next to the thermal wall has width and depth of $2000 \mathrm{~nm}$, and length of either 625 or $1250 \mathrm{~nm}$, corresponding to either 5 or 10 continuum cells. In the larger DSMC simulation the number of particles 


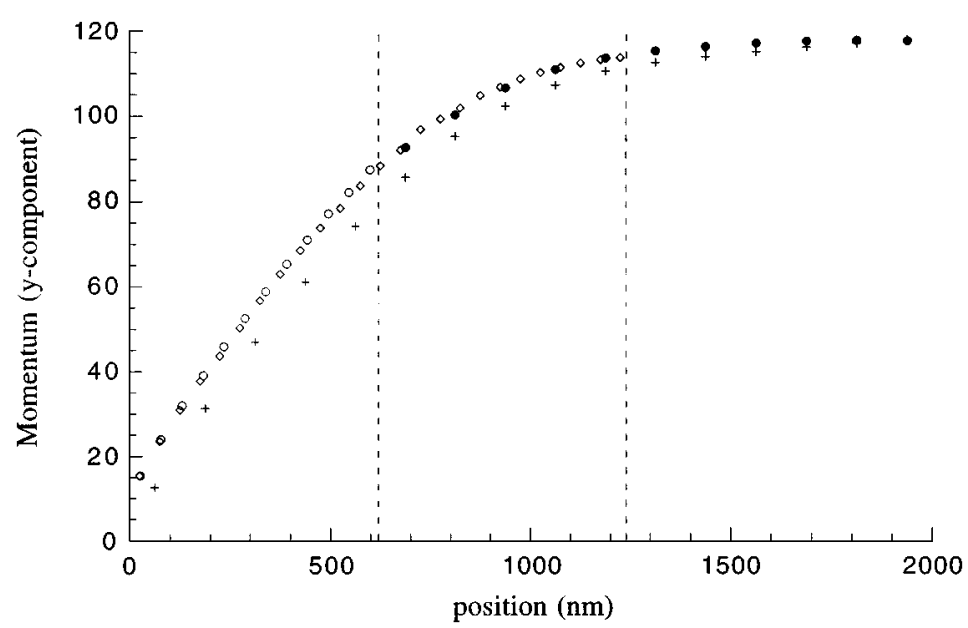

FIG. 9. Momentum density parallel to the wall versus position for the Rayleigh problem. AMAR run with 5-cell DSMC region $(\bigcirc)$; AMAR run with 10-cell DSMC region $(\diamond)$; and purely continuum with no DSMC run $(+)$. Open symbols DSMC data; filled symbols AMAR continuum data. Dashed lines indicate the location of the particle/continuum interface for the AMAR runs.

remains steady at about $2 \times 10^{6}$. For comparison, a calculation with only the Navier-Stokes solver and no DSMC region is also evaluated. Since the flow is entirely due to viscous drag, an Euler AMAR is not considered.

Figure 9 shows the component of momentum density parallel to the wall at $t=7.0 \times$ $10^{-9} \mathrm{~s}$. Note that there is good agreement between the two AMAR runs. The continuum solver uses no-slip boundary conditions at the thermal wall and thus fails to capture the Knudsen velocity slip at the wall. The slip length, that is, the distance within the wall at which the velocity extrapolates to zero, is $69 \mathrm{~nm}$, approximately one mean free path as expected from kinetic theory [2]. The normal component of momentum density is shown in Fig. 10. Because the flow in this direction is relatively weak, fluctuations are noticeable in the data points within the DSMC regions. Again, the two AMAR runs are in agreement and differ significantly from the purely continuum run.

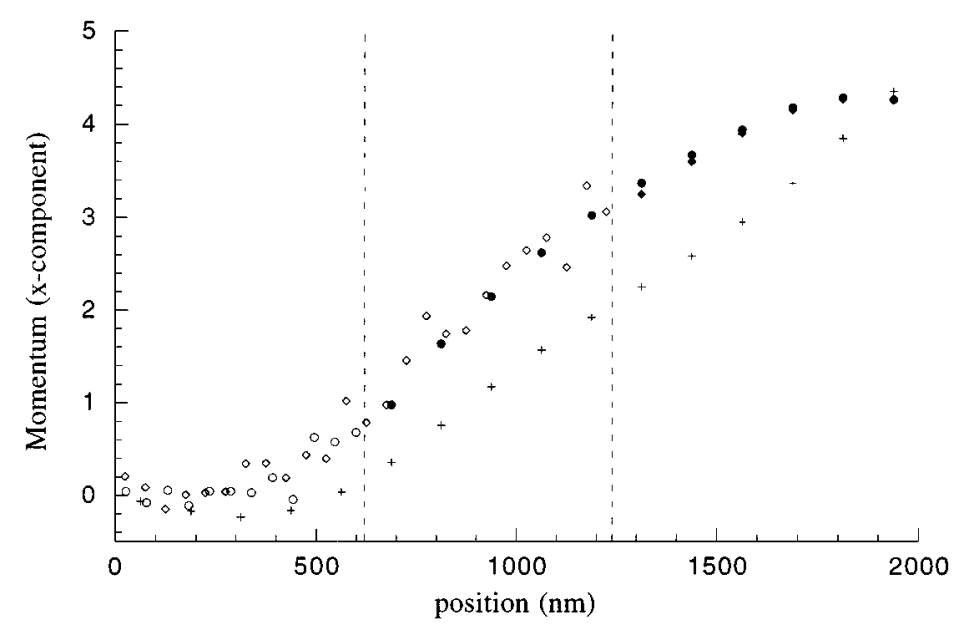

FIG. 10. Normal momentum density versus position for the Rayleigh problem. See Fig. 9 for legend. 


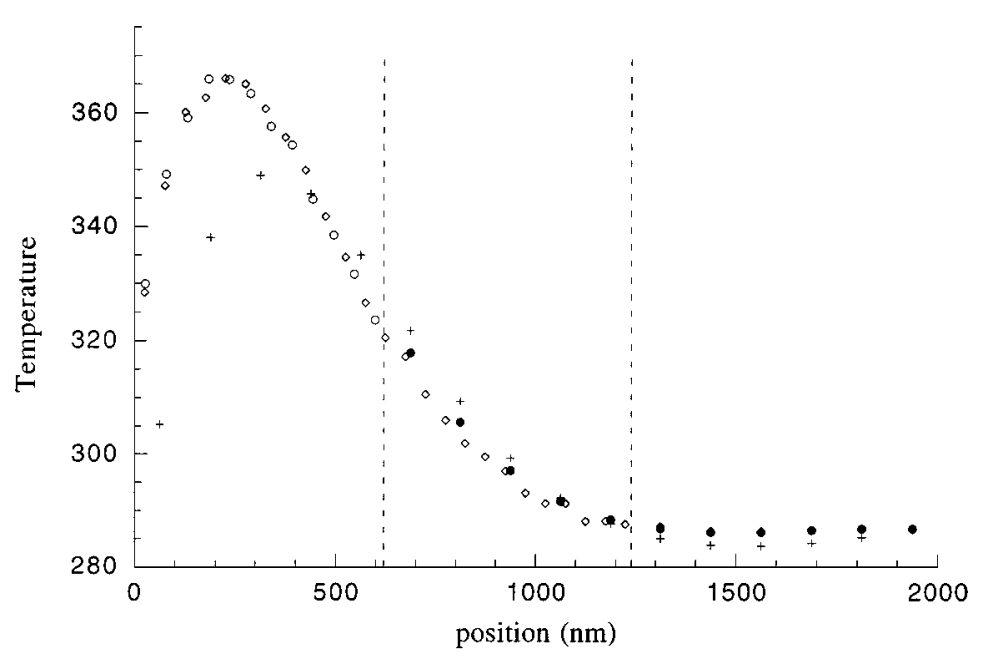

FIG. 11. Temperature versus position for the Rayleigh problem. See Fig. 9 for legend.

The flow away from the wall is due to the pressure gradient that develops when the temperature rises due to viscous heating (Fig. 11). The AMAR runs reproduce the Knudsen temperature jump at the wall. The distance within the wall at which the temperature profile extrapolates to the temperature of the wall is $120 \mathrm{~nm}$, approximately $\frac{15}{8}$ mean free paths as expected from kinetic theory [2]. Finally, the density profiles, shown in Fig. 12, indicate that a significant quantity of gas moves away from the wall. At the DSMC/Navier-Stokes interface, the Chapman-Enskog breakdown parameter has maximum values of $B=0.20$ and 0.15 for the 5 cell and 10 cell DSMC regions, respectively.

The difference between the AMAR results and the purely continuum results shown in Figs. 9-12 is primarily due to the Knudsen layer at the thermal wall. In order to investigate how thin a DSMC layer could still describe the wall conditions quantitatively, an AMAR run with a $1 \times 16 \times 16$ cell DSMC region was analyzed. The temperature profile in Fig. 13 shows that this thin particle layer captures the correct temperature jump but not the entire profile. At the DSMC/Navier-Stokes interface, the maximum of Chapman-Enskog breakdown

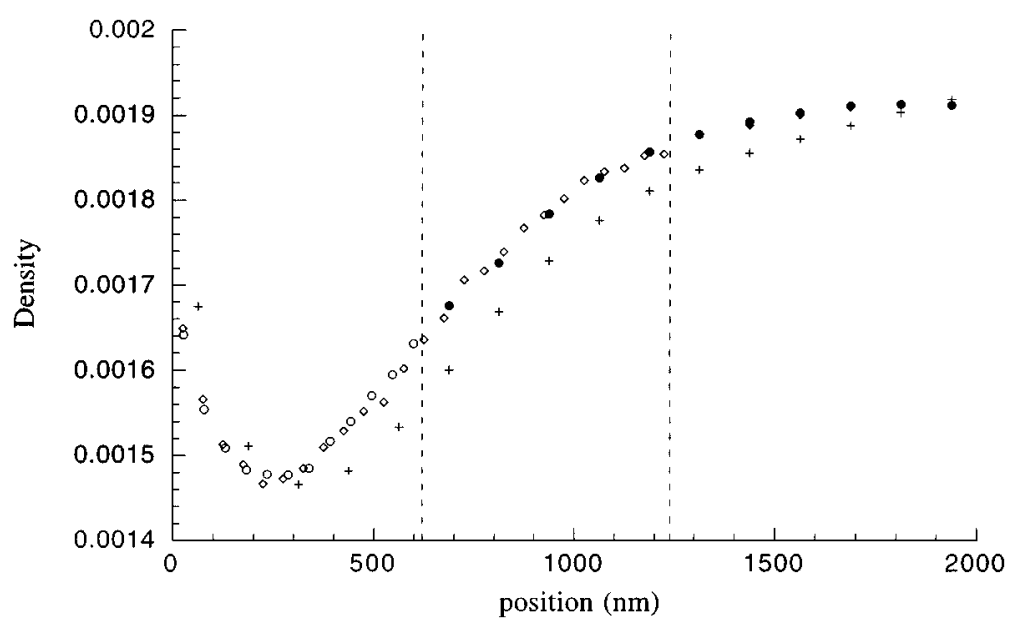

FIG. 12. Density versus position for the Rayleigh problem. See Fig. 9 for legend. 


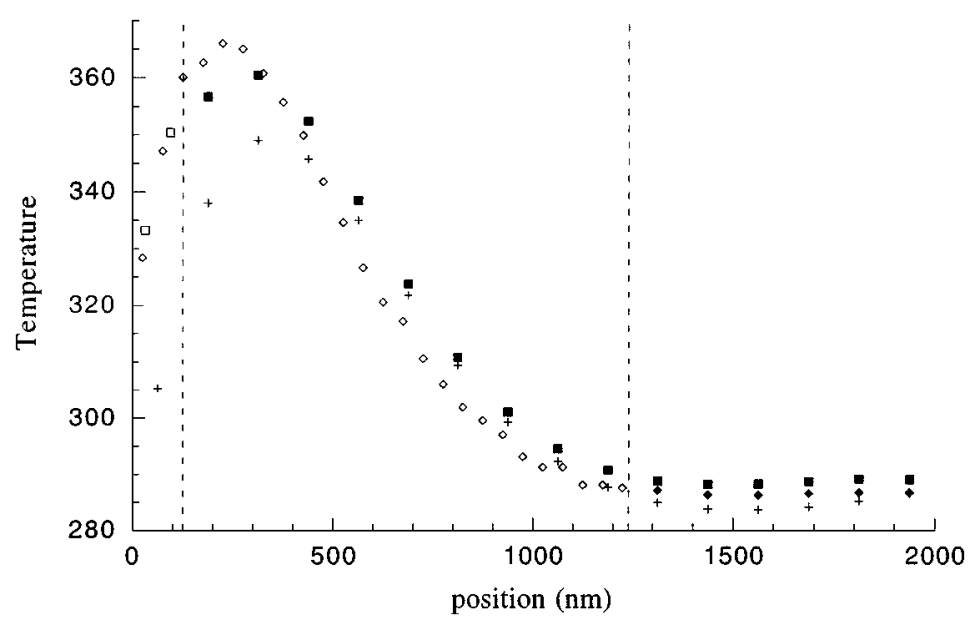

FIG. 13. Temperature versus position for the Rayleigh problem. AMAR run with 1-cell DSMC region ( $\square$ ); other symbols and lines as in Fig. 9.

parameter is $B=0.50$, which further indicates that the DSMC/continuum interface is too close to the wall.

\subsection{Flow Past a Sphere}

As a final example, flow past a microscopic object was considered. The body is a sphere held at the reference temperature and fixed at the center of the system (Fig. 4c). Inflow conditions are Mach 1 flow at the reference density and temperature. The continuum solver uses characteristic outflow boundary conditions with no diffusive fluxes. Periodic boundary conditions are applied in the other directions. While the flow is axially symmetric for this simple body, the calculation is fully three dimensional to demonstrate AMAR's capacity to simulate large scale flows.

The continuum solver uses two grids: a fine mesh embedded within a coarse mesh. The latter spans the entire system and contains $32 \times 32 \times 32$ coarse cells covering the system size of $8000 \mathrm{~nm}$ in each direction. The fine mesh covers a cube of length $4000 \mathrm{~nm}$ located at the center of the system. The DSMC region is a cube of length $1000 \mathrm{~nm}$ embedded in the center of the fine mesh. The DSMC region contains some $4 \times 10^{5}$ particles and occupies less than $0.2 \%$ of the total volume; see Fig. 14.

For both the continuum grid cells and the DSMC cells, the fractional volume occupied by the sphere is computed by a recursive method (Fig. 15). A cell is bisected in each direction; each corner of these subcells lies either inside or outside the body. If all corners are occupied (or empty), the fractional occupied volume for that subcell is one (or zero). Subcells which are partially occupied are further subdivided and this recursion continues until the desired accuracy is obtained. When the deepest level of recursion is reached, a subcell's occupied volume is estimated from the number of occupied corners.

The diameter of the sphere is $5 \lambda_{0}(312 \mathrm{~nm})$ thus $\mathrm{Kn}=0.2$. For Mach number $\mathrm{Ma}=1$ the Reynolds number is $\operatorname{Re}=8.24$. For these Knudsen and Reynolds numbers solutions of the Boltzmann equation predict a drag coefficient of $C_{D}=1.95$ when Ma $\ll 1$ [39]. A standing ring-eddy forms behind the sphere at $\operatorname{Re}>24$ [40] but since this numerical experiment is well below the critical Reynolds number vortices are not expected to form. 


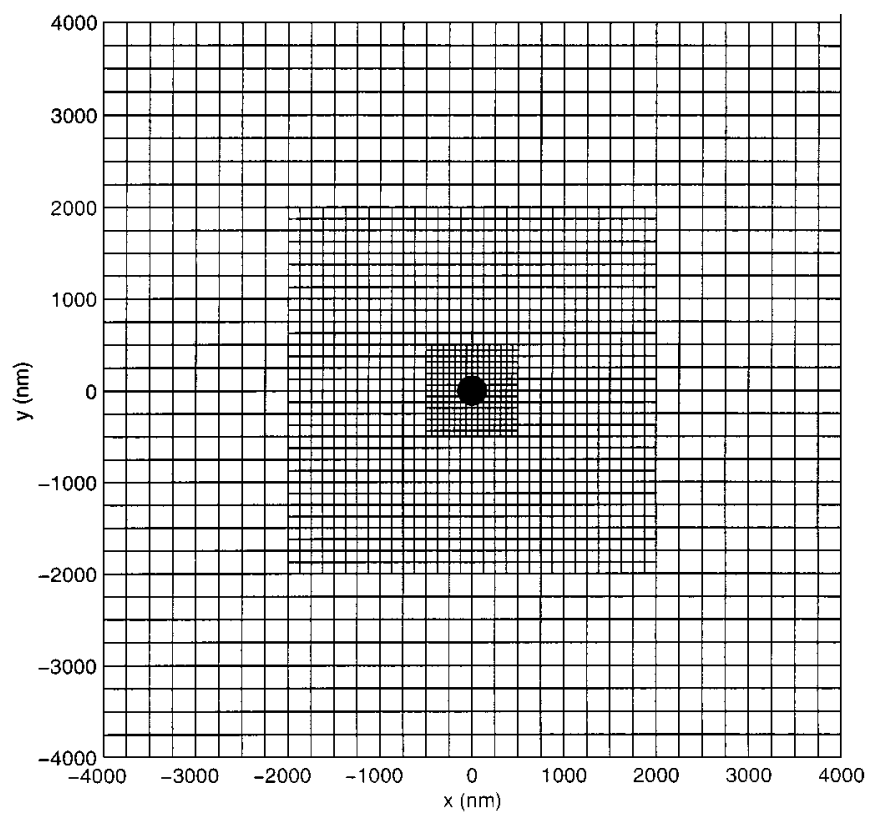

FIG. 14. Grids used in the simulation of flow past a sphere. Two outer grids used by Navier-Stokes solver; inner grid by DSMC. A finer subgrid, used within DSMC to select collision partners, is not shown.

Figure 16 shows the temperature contours for a cross-section through the center of the system; Fig. 17 is a blowup of the DSMC region. These and other profiles confirm that the flow is axially symmetric with little geometric distortion due to the rectangular grid. Irregularities in the contours are primarily due to statistical fluctuations in the DSMC region and finite grid resolution in the continuum region. The maximum value of the ChapmanEnskog breakdown parameter is $B=0.15$, indicating that the Navier-Stokes equations are accurate at the DSMC/continuum interface.

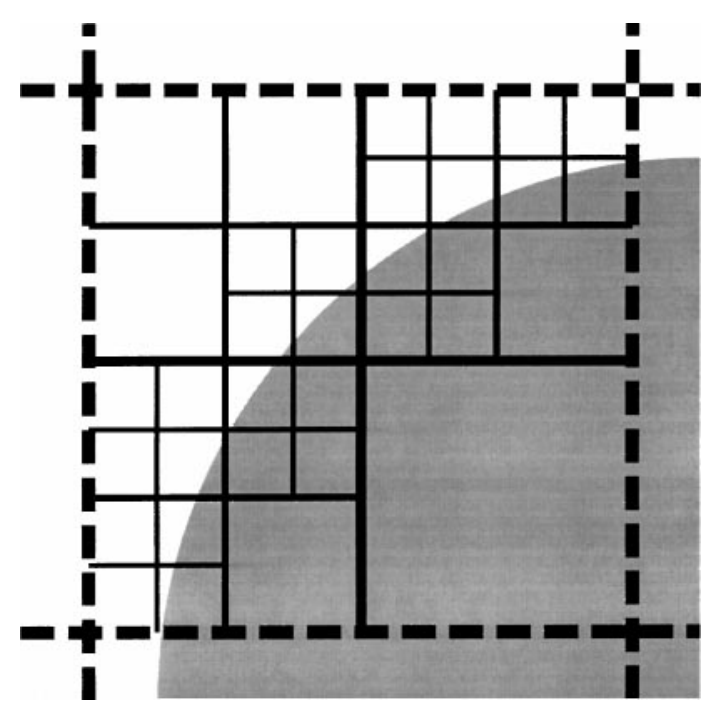

FIG. 15. Recursive method used to calculate the occupied volume of cells near the object. 


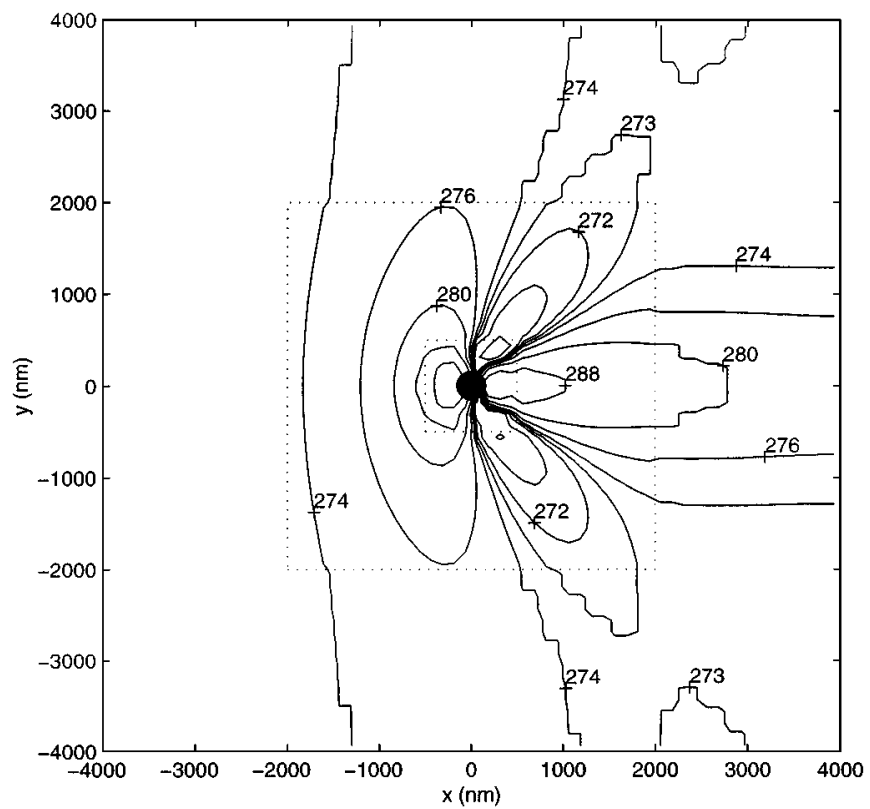

FIG. 16. Temperature contours in a centered cross-section for Mach 1 flow past a sphere. Contour levels are $\pm(1,2,4, \ldots) \mathrm{K}$ about the reference temperature of $273 \mathrm{~K}$. The dotted lines delineate the coarse/fine grid interface and the fine/DSMC grid interface.

For comparison, the temperature contours from a run with a 1500-nm DSMC region (over three times the volume) are shown in Fig. 18. There is close agreement with Fig. 16 showing that the 1000-nm DSMC region is sufficiently large, as was expected given the smallness of the breakdown parameter. The measured drag coefficient is $C_{D}=3.11$ for both runs,

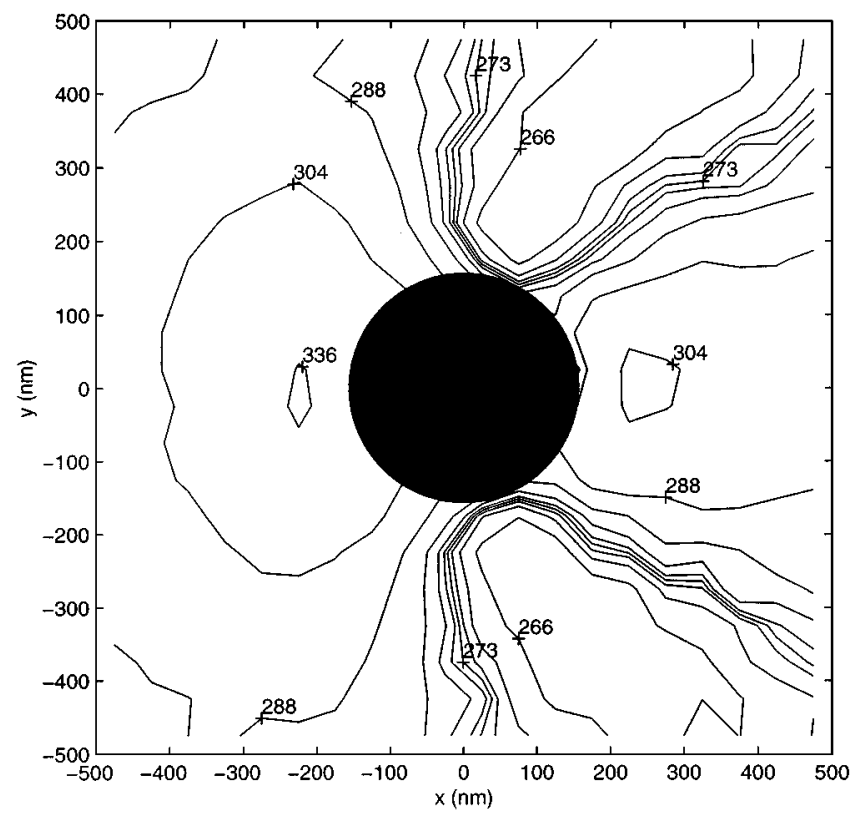

FIG. 17. Temperature contours, as in Fig. 16, but within the DSMC region. 


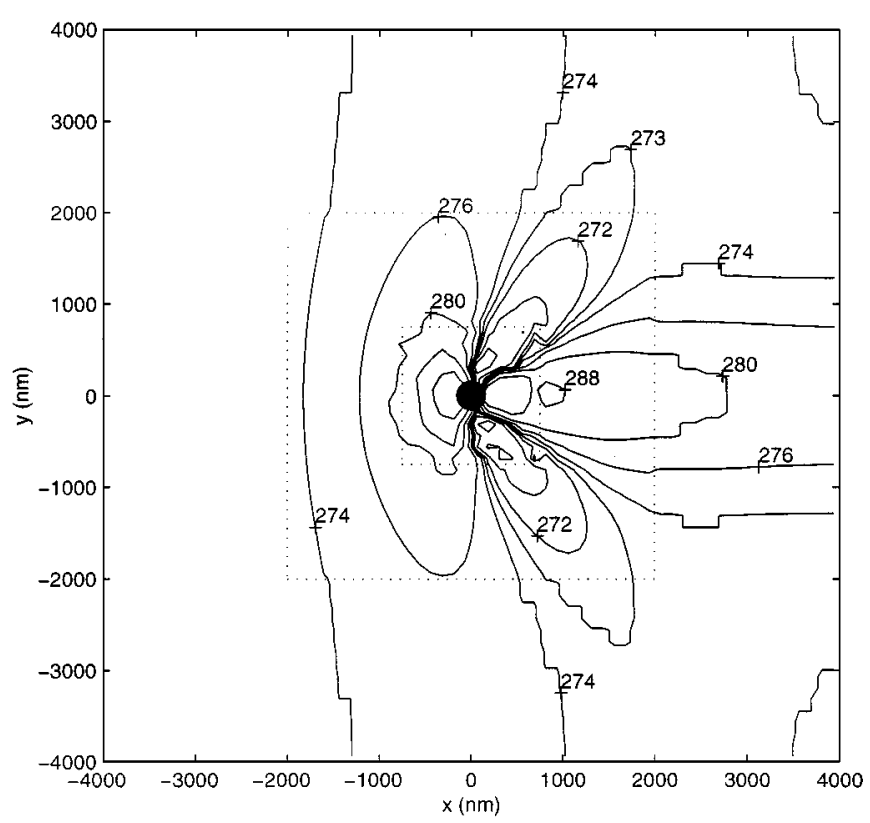

FIG. 18. Temperature contours, as in Fig. 16, but for a run with a 1500-nm DSMC region.

which is in good agreement with Bird's DSMC demonstration program. Finally, note that simulating the entire system using DSMC would require 200 million particles; a calculation of this magnitude is barely within the reach of today's largest supercomputers. The AMAR results presented here were obtained in a few hours on a DEC Alpha workstation.

\section{CONCLUDING REMARKS}

In the demonstrations given here the location of the interface between particle and continuum algorithms is fixed initially. While for some problems the suitable interface location may be known a priori, a more general approach is to have the simulation adaptively determine where to use each algorithm. The impulsive piston (Subsection 5.2) is a good example of a problem where an adaptive interface would be useful, namely the DSMC region should move with the shock keeping the wave front inside it. After the shock passes a given location, cells can revert to the continuum algorithm, thus making the program more efficient by minimizing the size of the DSMC region.

To implement an adaptive interface, a criterion that indicates the breakdown of the continuum formulation is required. Besides the breakdown parameter for the Chapman-Enskog distribution [34], several similar criteria have been proposed [41-43], and some have been implemented in DSMC/Euler hybrids $[18,44]$. Such an adaptive AMAR code is being developed and breakdown criteria will be evaluated for different physical situations.

In adaptive mesh refinement each grid level advances with its own time step, the coarsest grid using the largest time step. Thus an AMR calculation can span several orders of magnitude in both length scale and time scale; however, the finest time scales are constrained by the finest length scales. In AMAR, the DSMC region uses a time step that is comparable to but smaller than that used by the finest continuum grid. Though DSMC is unconditionally stable the method is only accurate when the time step is a fraction of the mean collision time. 
Both AMR and AMAR are useful for problems that span many time scales because a small time step is used only in those regions that require high resolution. When these regions occupy a small fraction of the system, as in the example of flow past a sphere (Subsection 5.4), most of the calculation advances at the larger time step. A variant of AMAR that uses the Schwarz alternating method [45] for computing steady flows is under investigation.

Further generalizations involve the implementation of the AMAR framework using other particle algorithms for applications at higher densities. The consistent Boltzmann algorithm (CBA) [23, 24], a generalization of DSMC to dense gases, can be used without modification to the coupling scheme. An additional generalization involves having particles interacting at a distance using molecular dynamics [46]. The modification to AMAR is that the fluxes of momentum and energy produced by the finite range interaction have to be computed in order to guarantee conservation. For dense gases and liquids, the velocity distribution for particles in the buffer cells is not known a priori but may be generated using the Schwarz alternating method.

Once these generalizations of the AMAR methodology have been developed there are a large number of applications that could more realistically simulate actual flows, particularly those that involve boundaries or interfaces. For example, flows near a wall could be represented by an atomistically rough boundary with the particle region embedded in the layer of cells near this surface. In that way the arbitrary stick or slip boundary conditions in the continuum representation could be replaced by a much more realistic one, possibly incorporating molecular surface scattering distributions [47]. One could also study how far boundary effects penetrate into the bulk fluid as a function of Reynolds number by increasing the width of the particle layer till the particle cells and continuum cells are equivalent.

Another possible application is the study of the Rayleigh-Taylor and Richtmyer-Meshkov instabilities where the interface between the two fluids would be represented by a few particle cell layers on each side. Besides giving a microscopically accurate representation of the interface, the spontaneous fluctuations in the particle region eliminate the need to use artificial perturbations to break the initial symmetry. A final example is the propagation of a crack in a solid [48]. At the tip of the crack a particle representation is required because phenomena occur on an atomistic scale; but further away, embedding the crack in an elastic continuum model is perfectly adequate. Using AMAR would avoid the huge number of particles required in a conventional molecular dynamics simulation to study the long time evolution of the crack since edge effects are eliminated when the continuum region is sufficiently large.

\section{ACKNOWLEDGMENTS}

The authors thank F. Alexander, D. Baganoff, V. Beckner, G. Bird, D. Goldstein, N. Hadjiconstantinou, D. Hash, R. Hornung, S. Kohn, and M. Lijewski for helpful discussions. The work was supported in part by National Science Foundation, Contract CTS-9711250; by the Applied Mathematical Sciences Program of the DOE Office of Mathematics, Information, and Computational Sciences, under Contract DE-AC03-76SF00098; and by the Department of Energy under Contract W-7405-ENG-48.

\section{REFERENCES}

1. F. Alexander, A. L. Garcia, and B. J. Alder, Direct simulation Monte Carlo for thin film bearings, Phys. Fluids 6, 3854 (1994). 
2. S. A. Schaff, and P. L. Chambre, in Fundamentals of Gas Dynamics (Princeton Univ. Press, Princeton, NJ, 1958).

3. S. Chapman and T. G. Cowling, The Mathematical Theory of Non-Uniform Gases (Cambridge Univ. Press, Cambridge, 1970).

4. D. Morris, L. Hannon, and A. L. Garcia, Slip length in a dilute gas, Phys. Rev. A 46, 5279 (1992).

5. K. Tibbs, F. Baras, and A. L. Garcia, Anomalous flow profile due to the curvature effect on slip length, Phys. Rev. E 56, 2282 (1997).

6. M. Malek Mansour, F. Baras, and A. L. Garcia, On the validity of hydrodynamics in plane poiseuille flows, Phys. A 240, 255 (1997).

7. D. Hash and H. Hassan, A Decoupled DSMC/Navier-Stokes Analysis of a Transitional Flow Experiment, AIAA Paper 96-0353 (1996).

8. F. de Jong, J. Sabnis, R. Buggeln, and H. McDonald, Hybrid Navier-Stokes/Monte Carlo method for reacting flow calculations, J. Spacecraft Rockets 29, 312 (1992).

9. D. C. Wadsworth and D. A. Erwin, One-Dimensional Hybrid Continuum/Particle Simulation Approach for Rarefied Hypersonic Flows, AIAA Paper 90-1690 (1990).

10. D. C. Wadsworth and D. A. Erwin, Two-Dimensional Hybrid Continuum/Particle Simulation Approach for Rarefied Hypersonic Flows, AIAA Paper 92-2975 (1992).

11. J. Eggers and A. Beylich, New algorithms for application in the direct simulation Monte Carlo method, Prog. Astro. Aero. 159, 166 (1994).

12. J. Bourgat, P. Le Tallec, and M. Tidriri, Coupling Boltzmann and Navier-Stokes equations by friction, J. Comput. Phys. 127, 227 (1996).

13. P. Le Tallec and F. Mallinger, Coupling Boltzmann and Navier-Stokes equations by half fluxes, J. Comput. Phys. 136, 51 (1997).

14. B. J. Alder, Search for a cheap molecular dynamics, in Monte Carlo and Molecular Dynamics of Condensed Matter Systems, edited by K. Binder and G. Ciccotti (Italian Physical Society, Bologna, 1996), p. 859.

15. B. J. Alder, Highly discretized dynamics, Phys. A 240, 193 (1997).

16. D. Hash and H. Hassan, A Hybrid DSMC/Navier-Stokes Solver, AIAA Paper 95-0410 (1995).

17. T. Lou, D. C. Dahlby, and D. Baganoff, A numerical study comparing flux-vector splitting for the NavierStokes equations with a particle method, J. Comput. Phys. 145, 489 (1997).

18. R. Roveda, D. B. Goldstein, and P. L. Varghese, A Combined Discrete Velocity/Particle Based Numerical Approach for Continuum/Rarefied Flows, AIAA Paper 97-1006 (1997).

19. G. A. Bird, Molecular Gas Dynamics and the Direct Simulation of Gas Flows (Clarendon, Oxford, 1994).

20. F. Alexander and A. Garcia, The direct simulation Monte Carlo method, Comput. Phys. 11, 588 (1997).

21. E. P. Muntz, Rarefied gas dynamics, Ann. Rev. Fluid Mech. 21, 387 (1989).

22. E. S. Oran, C. K. Oh, and B. Z. Cybyk, Direct simulation Monte Carlo: Recent advances and applications, Annu. Rev. Fluid Mech. 30, 403 (1998).

23. F. J. Alexander, A. L. Garcia, and B. J. Alder, A consistent Boltzmann algorithm, Phys. Rev. Lett. 74, 5212 (1995).

24. F. J. Alexander, A. L. Garcia, and B. J. Alder, The consistent Boltzmann algorithm for the van der Waals equation of state, Phys. A 240, 196 (1997).

25. F. J. Alexander, A. L. Garcia, and B. J. Alder, Cell size dependence of transport coefficients in stochastic particle algorithms, Phys. Fluids 10, 1540 (1998).

26. M. Fallavollita, D. Baganoff, and J. McDonald, Reduction of simulation cost and error for particle simulations of rarefied flows, J. Comput. Phys. 109, 30 (1993)

27. G. Chen and I. Boyd, Statistical error analysis for the direct simulation Monte Carlo technique, J. Comput. Phys. 126, 434 (1996).

28. D. A. Anderson, J. C. Tannehill, and R. H. Pletcher, Computational Fluid Mechanics and Heat Transfer (Hemisphere, New York, 1984).

29. J. Saltzman, An unsplit 3D upwind method for hyperbolic conservation laws, J. Comput. Phys. 115, 153 (1994). 
30. M. Berger and J. Oliger, Adaptive mesh refinement for hyperbolic partial differential equations, J. Comput. Phys. 53, 484 (1984).

31. M. Berger and P. Colella, Local adaptive mesh refinement for shock hydrodynamics, J. Comput. Phys. 82, 64 (1989).

32. J. Bell, M. Berger, J. Saltzman, and M. Welcome, Three-dimensional adaptive mesh refinement for hyperbolic conservation law, SIAM J. Sci. Comput. 15, 127 (1994).

33. A. S. Almgren, J. B. Bell, P. Colella, L. H. Howell, and M. L. Welcome, A conservative adaptive projection method for the variable density incompressible Navier-Stokes equations, J. Comput. Phys. 142, 1 (1998).

34. A. L. Garcia and B. J. Alder, Generation of the Chapman-Enskog distribution, J. Comput. Phys. 140,66 (1998).

35. M. Malek Mansour, A. L. Garcia, G. Lie, and E. Clementi, Fluctuating hydrodynamics in a dilute gas, Phys. Rev. Lett. 58, 874 (1987).

36. G. A. Bird, personal communication, 1996.

37. D. B. Goldstein, personal communication, 1998.

38. L. D. Landau and E. M. Lifshitz, Fluid Mechanics (Pergamon, Oxford, 1959).

39. S. Takata, Y. Sone, and K. Aoki, Numerical analysis of a uniform flow of a rarefied gas past a sphere on the basis of the Boltzmann equation for hard-sphere molecules, Phys. Fluids 5, 716 (1993).

40. G. K. Batchelor, An Introduction to Fluid Dynamics (Cambridge Univ. Press, Cambridge, 1967).

41. G. Bird, Breakdown of translational and rotational equilibrium in gaseous expansions, Am. Inst. Aero. Astro. J. 8, 1998 (1970).

42. I. D. Boyd, G. Chen, and G. V. Candler, Predicting failure of the continuum fluid equations in transitional hypersonic flows, Phys. Fluids 7, 210 (1995).

43. C. D. Levermore, Wm. J. Morokoff, and B. T. Nadiga, Moment realizability and the validity of the NavierStokes equations for rarefied gas dynamics, Phys. Fluids 10, 3214 (1998).

44. S. Tiwari and A. Klar, Coupling of the Boltzmann and Euler equations with adaptive domain decomposition procedure, J. Comput. Phys. 144, 710 (1998).

45. N. Hadjiconstantinou and A. Patera, Heterogeneous atomistic-continuum representations for dense fluid systems, Int. J. Mod. Phys. C 8, 967 (1997).

46. M. P. Allen and D. J. Tildesley, Computer Simulation of Liquids (Clarendon, Oxford, 1987).

47. T. E. Wenski, T. Olson, C. T. Rettner, and A. L. Garcia, Simulations of air slider bearings with realistic gas-surface scattering, J. Tribology 120, 639 (1998).

48. F. F. Abraham, D. Brodbeck, R. A. Rafey, and W. E. Rudge, Instability dynamics of fracture: A computer simulation investigation, Phys. Rev. Lett. 73, 272 (1994). 